\title{
Als Mathelehrer noch Rechenmeister waren
}

\author{
Erzählen und Rechnen in Textaufgaben der frühen Neuzeit
}

\author{
Edith Feistner und Alfred Holl
}

\begin{abstract}
In Zeiten einer Hochkonjunktur der MINT-Fächer ist es kaum vorstellbar, dass Mathematik lange Zeit gar kein normales Schulfach war und auch kein Bestandteil des Abiturs. Umso mehr lohnt sich ein Blick in die Geschichte der Mathematisierung der Gesellschaft, die sich am Übergang vom Spätmittelalter zur frühen Neuzeit in Gestalt der Rechenkunst (ars arithmetica) Bahn bricht: Parallel zur Etablierung der Stadtkultur mit Handel, Gewerbe und Verwaltung kommt es zu einer sich von Spanien, Italien und Byzanz aus über ganz Europa erstreckenden Verbreitung der Kulturtechnik des Rechnens.
\end{abstract}

Die Vermittlung der Kulturtechnik des Rechnens lag in den Händen von Rechenmeistern, die in Städten eigene Rechenschulen betrieben. Rechenmeister mussten das Bürgerrecht haben, um die Eröffnung einer Schule beantragen zu können, und sie mussten verheiratet sein, um ein der Schule angegliedertes Internat zu führen. Sie waren wichtige Mittler für die schulische und universitäre Verankerung der Mathematik. Aus akademischer Sicht galten sie zunächst freilich als Handwerker, die bloß anwendungsorientiertes „Kaufmannswissen“ weitergaben und daneben oft genug die Aufgabe einfacher „Schulhalter" übernahmen. Sie absolvierten ihre Ausbildung bei einem Meister in Form einer Lehre, obwohl ein zunehmender Anteil unter ihnen bereits eine höhere Schulbildung mitbrachte und über den Horizont der Kaufmannsmathematik hinauszusehen in der Lage war. Nicht zufällig dokumentiert sich ein durchaus elitäres corporate identity-Bewusstsein unter Rechenmeistern und ihren Familien: Man kannte einander - auch über Stadtgrenzen hinweg und knüpfte „dynastische“ Bande.

Die von besonders kompetenten, ambitionierten Rechenmeistern verfassten Rechenbücher waren als Drucke ein verbreiteter Bestandteil der Unterrichtspraxis. Sie wandten sich an Lernende unterschiedlichen Alters, auch an Erwachsene, die sich im Selbststudium beruflich weiterbildeten. Als didaktisches Mittel der Wahl dominierten neben reinen Umrechnungsaufgaben vor allem Textaufgaben, die Fallgeschichten erzählten. So ließ sich anschaulich durchexerzieren, wo, wann und wozu man $\mathrm{zu}$ rechnen hatte, bevor der mit Ist die Frag eingeleitete Anschluss der Rechenaufgabe an die Erzählung hergestellt wurde und die Angabe des richtigen Ergebnisses, das $\mathrm{Fa}$ cit, folgte. Das Erzählen war aber auch der Schlüssel zum Tor, das aus der Kaufmannsmathematik herausführt: Der erfahrene Aufgabensteller wusste, dass Fallgeschichten als „Übersetzungen“ mathematisch-formaler Rechenaufgaben in raumzeitliche Wirklichkeit(en) mitnichten an alltagspraktische Konstellationen gebunden waren. Gerade im 17. Jahrhundert, als die in Deutschland mit dem 15. Jahr- hundert beginnende Geschichte der Rechenbuchdrucke ihren Höhepunkt erreichte, begegnen uns hier immer wieder Fallgeschichten, die weit über die Welt von Handel und Gewerbe hinausgehen, die verschiedensten Lebensund Wissensbereiche umfassen, ja überhaupt nur mathematisch denkbar sind. Am Beispiel von Regensburger Rechenbüchern ist ein interdisziplinäres Kooperationsprojekt zwischen der Universität Regensburg und der TH Nürnberg auf Spurensuche in die Geschichte gegangen.

\section{Rechenmeister-Biografien}

Die Geschichte der Regensburger Textaufgaben beginnt mit dem Benediktiner Fridericus Amann (ca. 1405-1465) aus dem Kloster St. Emmeram. Mit seinem Namen eng verbunden ist eine um 1450 handschriftlich überlieferte Sammlung von 354 Textaufgaben in deutscher und lateinischer Sprache, die Practica des Algorismus Ratisbonensis. Einen Algorismus nannte man im Mittelalter eine Anleitung zum Rechnen mit indisch-arabischen Zahlen; die Bezeichnung ist der latinisierte Name des persischen Gelehrten Mohammed ben Musa al-Khwarizmi (ca. 780 bis ca. 850), auf den auch der heutige Ausdruck Algorithmus für eine automatisierbare Verarbeitungsvorschrift zurückgeht. Diese Sammlung übte einen enormen Einfluss auf die ersten gedruckten Rechenbücher aus, die andernorts, nach den ersten Anfängen um 1475, ab 1525 regelrecht aus dem Boden schossen. Am berühmtesten sind wohl die Werke des in Annaberg im Erzgebirge tätigen Adam Ries (1492-1559), auf den der Spruch „Das macht nach Adam Ries..." zurückgeht.

Regensburg jedoch erlebte nach Fridericus Amann „mathematisch ruhige“ 1 oo Jahre. Der als Astronom und Kartograf bekannte Peter Bienewitz (1495/1501-1552), latinisiert zu Apian, hatte von 1521 bis 1526, zwischen Studium in Wien und Berufung auf einen Mathematiklehrstuhl an der 1572 gegründeten bayerischen Landes- 


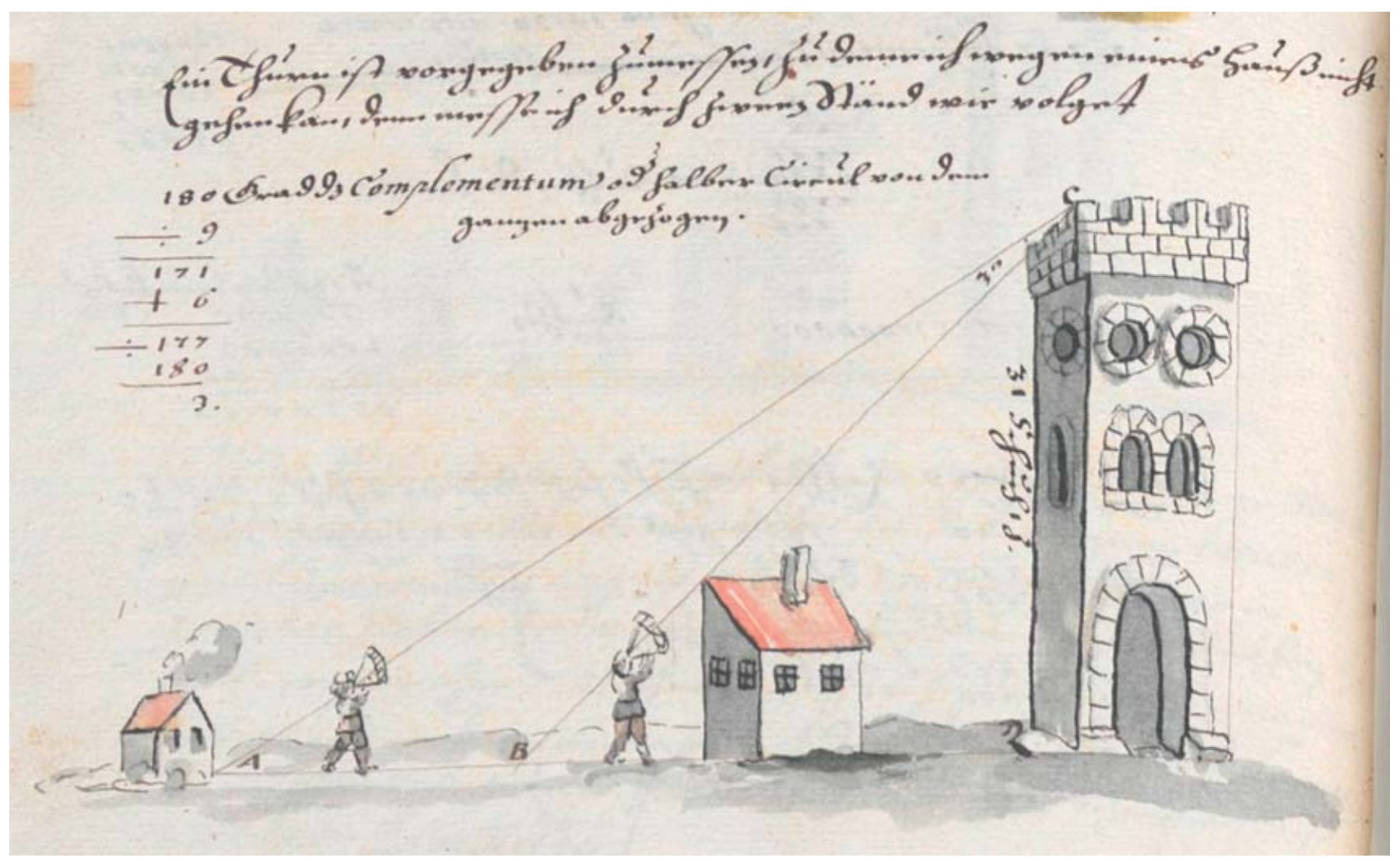

Höhenbestimmung eines Turms (Georg Wendler, Memorialbuch. Bayerische Staatsbibliothek München, Cgm 3788, 155v)

Ein Thurn ist vorgegeben zumessen, zu deme ich wegen eines Hauß nicht gehen kan, dene messe ich durch zween Ständ. Wegen eines Hauses, das „im Weg“ steht, kann man die Entfernung eines Messpunktes vom Turm nicht ermitteln. Deshalb braucht man zwei Messpunkte, deren Abstand man kennt. Von jedem Messpunkt aus wird mit einem Quadranten der Winkel bestimmt, unter dem die Zinnen des Turms gegen die Horizontale erscheinen.

universität in Ingolstadt, einen flüchtigen Kontakt zu Regensburg. In einer beruflich eher ungewöhnlichen Kombination war er zugleich Universitätsmathematiker und Rechenbuchautor (Eyn newe vnnd wolgegründte underweysung aller Kauffmannß Rechnung, Ingolstadt 1527). Unter seinem Einfluss entstand 1524 eine (2013 entdeckte) Handschrift (Herzogliches Georgianum München, $4^{\circ}$ Ms 50), die explizite Bezüge zu Regensburg enthält. Ansonsten gab es zu dieser Zeit in Regensburg keine Autoren von Rechenbüchern.

Das erste gedruckte Regensburger Rechenbuch, die Arithmetica des möglicherweise in Amberg ausgebildeten Johann Kandler (ca. 1530-1600) aus Böhmisch Budweis, datiert erst 1578 . Es wurde 1608 zum dritten Mal aufgelegt. Dazu sind zwei bisher unbeachtete Rechenhandschriften aus den 159 oer Jahren erhalten (Bayer. Staatsbibliothek München, Cgm 4143 und Cgm 4144), die zweifellos eine unterrichtspraktische Verwendung dieses Buchs bezeugen. Der Rechenschüler, der sie schrieb, hieß Bartholomäus Fuchs (1578-1653). Er arbeitete später unter anderem als Substitut des Regensburger Stadtschreibers. Im Jahre 1609 kam der aus einer traditionsreichen Nürnberger Dynastie stammende Anton Neudörffer (1571-1628) aus unbekannten Gründen nach Regensburg. Er veröffentlichte die Neuauflagen seines Rechenbuchs Anweisung in die Arithmetic weiterhin in Nürnberg. Der einzige Regensburger Rechenmeister, der ihn würdigte, war Georg Wendler (s. u.). In einer handschriftlichen Bearbeitung von Aufgaben aus verschiedenen Rechenbüchern widmete er ihm gleich zu Anfang 215 Blatt. Darunter finden sich - 2014 neu entdeckt - Teile von Neudörffers angekündigter, aber nie gedruckter Grosser Arithmetic.

Ab 1631 wurde Georg Wendler (1619-1688) auf Kosten der Stadt Regensburg in Nürnberg ausgebildet. Seit 1647 arbeitete er in Regensburg. Die Bayerische Staatsbibliothek München besitzt zwei hervorragende illustrierte Handschriften aus der Mitte des 17. Jahrhunderts, in denen er seinen mathematischen Interessen frönt. Die erste (Cgm 3788) enthält auch die Aufgaben seiner Nürnberger Rechenmeisterprüfung von 1646 und sein Rechenmeisterzeugnis. In der zweiten Handschrift (Analysis vel resolutio betitelt, Cgm 3789) hat er Textaufgaben aus verschiedenen Rechenbüchern gelöst, sogar solche von Kandler und Neudörffer. 1667 erschien Wendlers Arithmetica practica als explizites Nachfolgewerk der Arithmetica von Johann Kandler, der dort in den Begleittexten hoch gerühmt wird.

Damit wurde eine eigenständige Regensburger Rechenbuch- und Rechenmeister-Tradition mit einem markanten corporate identity-Bewusstsein begründet, die Georg Heinrich Paritius (1675-1725) fortsetzte. Paritius, dessen Ausbildungsgang unbekannt ist, veröffentlichte 1706 sein erstes Rechenbuch, die Praxis Arithmetices. 
Danach folgten zahlreiche kaufmannsmathematische Werke für unterschiedliche Zielgruppen. Über seinen Lehrerberuf hinaus war es Paritius wichtig, einem zeitgenössischen Netzwerk von Rechenmeistern mit höheren Ansprüchen anzugehören, nämlich der 1690 gegründeten Kunst-Rechnungs-liebenden Societät, der heutigen „Mathematischen Gesellschaft in Hamburg". Ein Kuriosum seines Lebens sind die 1718 aktenkundig gewordenen Auseinandersetzungen mit seiner zweiten Frau (Stadtarchiv Regensburg), die seinen Ruf wohl so schädigten, dass er um 1720 begann, heute historisch wertvolle, lokalgeschichtliche Werke zu erstellen - als alternative Einnahmequelle.

\section{Textaufgaben-Beispiele}

Kaufmannswesen: Oberpfälzisch-fränkische

Schiffsreisegesellschaft

Bei einer Reise von Regensburg nach Wien mieten mehrere Personen gemeinsam ein Schiff. Die ersten Reisenden, fünf Regensburger Bürger, treffen gleich zu Anfang mit dem Schiffseigner eine Absprache, wie sich der Fahrpreis pro Person verringern soll, wenn später noch mehr Fahrgäste mitfahren wollten. Der Beitrag von vier hinzukommenden Nürnberger Kaufleuten wird demgemäß je zur Hälfte auf die ursprünglichen Reisenden, die jetzt weniger zahlen müssen, und den Schiffseigner verteilt, sodass alle Reisenden den gleichen Fahrpreis bezahlen (Kandler, Arithmetica, Kiii-iii', Nr. 24). Da der Schiffseigner von dem Betrag, den jeder der vier Nürnberger beisteuert, nur die Hälfte bekommt, muss er umsonst $4 / 2=2$ Personen zusätzlich für die ursprünglich angesetzten $16 \mathrm{fl}$ transportieren, insgesamt also 7. Diese Tatsache bestimmt den neuen Fahrpreis pro Person: $16 / 7 \mathrm{fl}=2 \mathrm{fl} 2 \mathrm{~B}$ mit der Umrechnung 1 Gulden $(\mathrm{fl})=7$ Schilling $(B)$. Insgesamt nimmt der Schiffman aber 9 Personen mit, sodass er $18 \mathrm{fl} 18 ß=20 \mathrm{fl} 4 \mathrm{~B}$ erhält.

\section{Sozialgeschichte: Bayerische Wirtshauskultur,}

\section{Anfang des 18. Jahrhunderts}

Paritius lässt drei lustige Brüder um die Wette trinken, allerdings beginnen sie gemeinsam, weil nur eine begrenzte Menge, ein Väßlein Sommer-Bier à 60 Köpff, zur Verfügung steht. Kopf ist übrigens die damals gängige Bezeichnung für die Maß. A würde das ganze Fass in 30 Stunden schaffen, B in 20 und C in 12 (Paritius, Neugemehrte Praxis Arithmetices, Teil 3, S. 198 f., Nr. 2). A ist der langsamste mit einer Trinkgeschwindigkeit von $2 \mathrm{Maß} / \mathrm{h}$, B hält die Mitte mit $3 \mathrm{Maß} / \mathrm{h}$, und $\mathrm{C}$ ist der schnellste mit $5 \mathrm{Maß} / \mathrm{h}$. Aufaddiert ergeben sich $10 \mathrm{Maß} / \mathrm{h}$, sodass das Fässlein in 6 Stunden leer wird und A 12, B 18, C 30 Maß bekommt.

\section{Literatur: Liebespaar am Quellbrünnlein}

Auf die Verbindungslinie zweier verschieden hoher italienischer Palazzi legt Neudörffer ein liebliches Quellbrünnlein, an dem sich nächtens zwei Verliebte aus den beiden Palatia treffen. Im gespielten Streit ob der zurückzulegenden unterschiedlichen Fußwege stellen sie fest, dass sie als Vögelein von ihren Zinnen aus zum Brunnen gleich weit zu fliegen hätten.
In Italia einer Villa hab ich mei= ner Zeit gesehen 2 schöner Palatia/ die stunden gerad gegen einander über/ dazwischen war auff der Erden ein liebliches Quellbrünnlein/ wel= ches zwey Liebe offtermals nächtlicher weile be= suchten/ vnnd sich darzu funden. Auff eine zeit thete sich die Jungfraw gegen jhrem Liebhaber schertzweise beschweren/ obwoln jhre Zinnen deß Pallasts nur 3/4 so hoch als die seine/ müste sie doch

70 Schritt jhme zu gefallen von Hauß auß meh= rers thun/ als Er/ biß sie zum Brünnlein käme/ der gibt darauff diese Antwort/ es were jhm zwar leid/ doch ob er wol 35 Schritt weniger zum Brunnen habe/ als jhre Zinnen schuch von der Erden/ dünck jhne doch die Zeit gar kurtz seyn/ biß er von seiner hohen Zinnen herab käme. Wünschte aber/ daß sie beyde Vögelein weren/ so hette eins so weit als das ander von jedes $\mathrm{Zin}=$ nen gerad zum LiebBrunnen zu fliegen. Ist die frag/ wie hoch jedes Zinnen schuch/ vnnd jedwe= ders Schritt zum Brünnlein?

(Neudörffer, Anweisung in die Arithmetic, S. 213, Nr. 68)

Zur Lösung wird hier die etwas merkwürdige - aber von Neudörffer offensichtlich intendierte - Gleichsetzung der Maße schuch und Schritt in Wendlers Bearbeitung (Cgm 3789, 102rv) herangezogen (normalerweise gilt ein Schritt als ungefähr zweieinhalb mal so lang wie ein Schuh oder Fuß).

Die Jungfraw J habe $x$ Schritte zum Quellbrünnlein zu gehen, der Liebhaber $L(x-70)$ Schritte. Ihre Zinnen sind $(x-70+35)$ hoch, seine $(x-70+35) \times(4 / 3)$. Die Hypotenusen („Flugstrecken“) der so entstehenden rechtwinkligen Dreiecke müssen gleich lang sein, also nach dem Satz von Pythagoras:

$$
\begin{aligned}
& \text { Wegstrecke }_{J}{ }^{2}+\text { Zinnenhöhe }_{J}{ }^{2} \\
& =\text { Wegstrecke }_{L}{ }^{2}+\text { Zinnenhöhe }_{L}{ }^{2}, \\
& x^{2}+(x-35)^{2}=(x-70)^{2}+(x-35)^{2}(4 / 3)^{2}, \\
& x^{2}-250 x+7525=0 .
\end{aligned}
$$

Die positive Lösung dieser quadratischen Gleichung liefert die Wegstrecke der Jungfraw mit $x=215$ Schritt; die negative Lösung (-35) ist irrelevant. Die restlichen GröBen sind damit leicht zu berechnen.

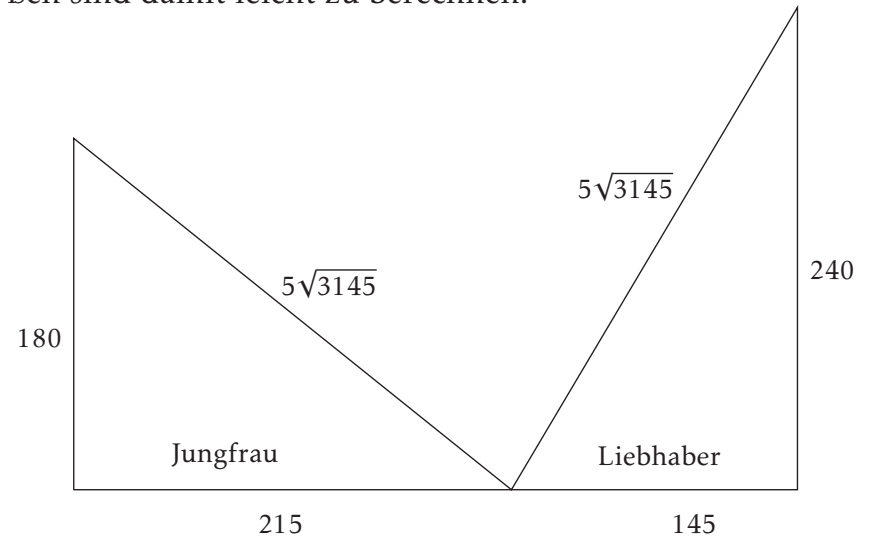


Fiktion: Raub der Ameiseneier

Ans Ende dieser Auswahl sei ohne Lösung eine schwierige Variante eines Aufgabentyps gestellt, der singulär bei Anton Neudörffer auftritt. Sie soll zu eigenem Nachdenken anregen, den Blick auf Buch und Online-Ausstellung lenken (s. u.) und zeigen, welch komplexe Sachverhalte mit trickreicher Anwendung von Mittelstufenmathematik gelöst werden können. Neudörffer selbst, der offenbar schon ein Gefühl für mathematische Ästhetik besitzt, kommentiert am Ende: wer aber den rechten vortheil nicht gebrauchet/dem werdens mühe machen/ wie schön die solutio auch ist.

Die Situation ist in wenigen Worten skizziert: Es geht um elf Ameisenhaufen, die alle unterschiedlich viele Ameiseneier beherbergen. Die Ameisen aus Haufen 1 bis 10 plündern nun Haufen 11 und holen sich daraus je so viele Eier, wie sie schon vorher hatten. Dann fallen die Ameisen aus Haufen 1 bis 9 und 11 über den Haufen 10 und rauben wieder so viele Eier, dass sich ihr jeweiliger aktueller Bestand verdoppelt. Das Verfahren setzt sich in gleicher Weise über die Haufen 9 bis 1 fort. Am Ende habe jeder der 11 Ameisenhaufen die gleiche Menge Eier, und es fragt sich, wie viele jeder am Anfang besaß. Jedes ganzzahlige Vielfache einer Lösung ist natürlich wieder eine Lösung, sodass Neudörffer nur die kleinste (PrimZahl) sucht (Neudörffer, Anweisung in die Arithmetic, S. 187, Nr. 186).

Textaufgaben als mathematisch-germanistische Fundgrube

Mathematik ist abstrakt, entgegen gängiger Rede aber keineswegs automatisch auch ,trocken'. Das dürften die vorgestellten Beispiele deutlich gezeigt haben. Gerade weil Mathematik abstrakt ist - sicher die abstrakteste unter allen Wissenschaften - und mathematische Gegenstände nur Zeichen sind, die es in der außermathematischen Wirklichkeit nirgends gibt, kann der mathematische Blick auch überall Anschluss und Zugang finden. Weil für Mathematik die Zahlen und nicht die Fakten außerhalb der Mathematik zählen, kann sie sogar über die Grenze zwischen Fakten und Fiktionen hinaus erzählen.

Die Textaufgaben in frühneuzeitlichen Rechenbüchern schöpfen dieses Potenzial zur Vermittlung mathematischen Wissens voll aus. Der Blick in die (Mathematik-) Geschichte kann also durchaus auch heute ein Schlüssel zur Mathematik sein. Nicht zuletzt aber bieten mathematische Textaufgaben aufgrund ihrer fast alle Lebens- und Wissensbereiche umfassenden Reichweite ein bemerkenswert vielfältiges Spektrum an Anknüpfungspunkten für interdisziplinäre kulturwissenschaftliche Fragestellungen. Mathematische Textaufgaben erlauben es, eine Brücke von großer Spannweite über die Grenze zwischen natürlicher und formaler Sprache hinweg zu schlagen: von Fragen der Mathematik(geschichte) über Fragen der Wissenschaftsgeschichte bis hin zur Germanistik.

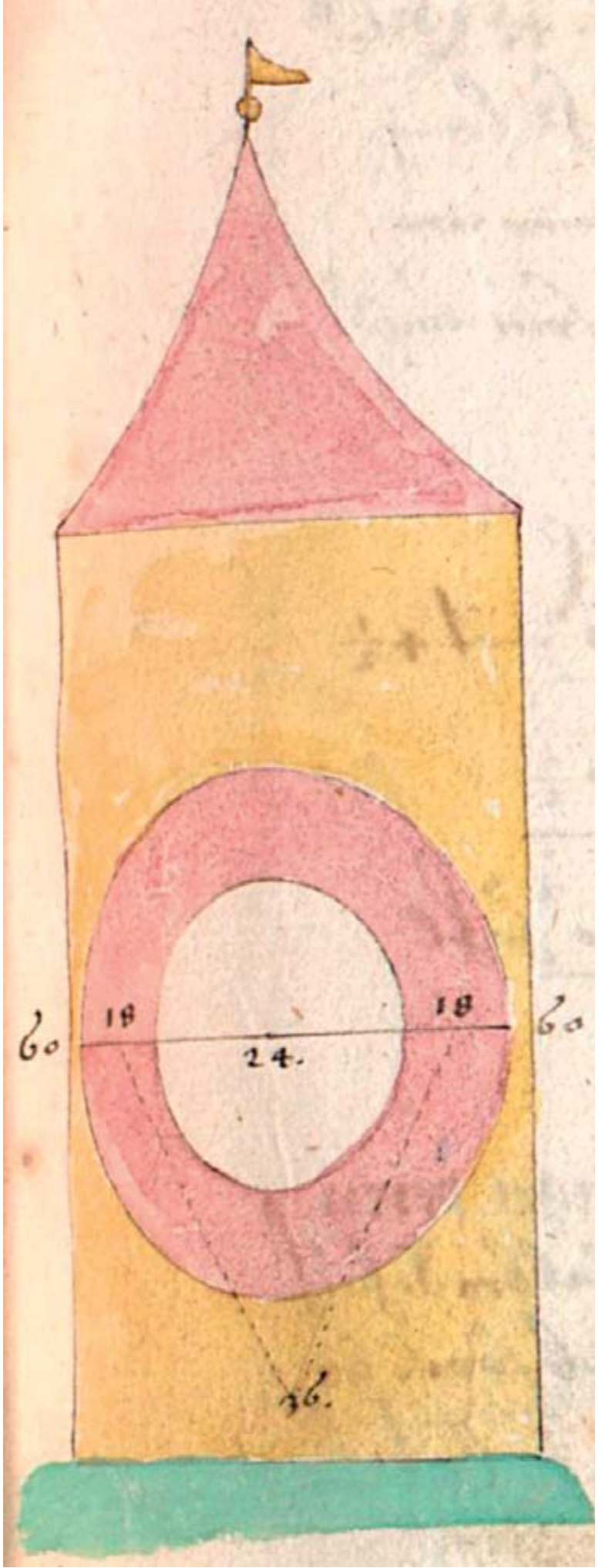

Georg Wendler, Ausschnitt aus Planskizze (Vgl. Abbildung auf S. 110)

So entstand ein interdisziplinäres, von der Regensburger Universitätsstiftung Pro arte gefördertes Kooperationsprojekt zwischen der Universität Regensburg und der TH Nürnberg. Die Staatliche Bibliothek Regensburg zeigte begleitend vom 6. Oktober bis 6. November 


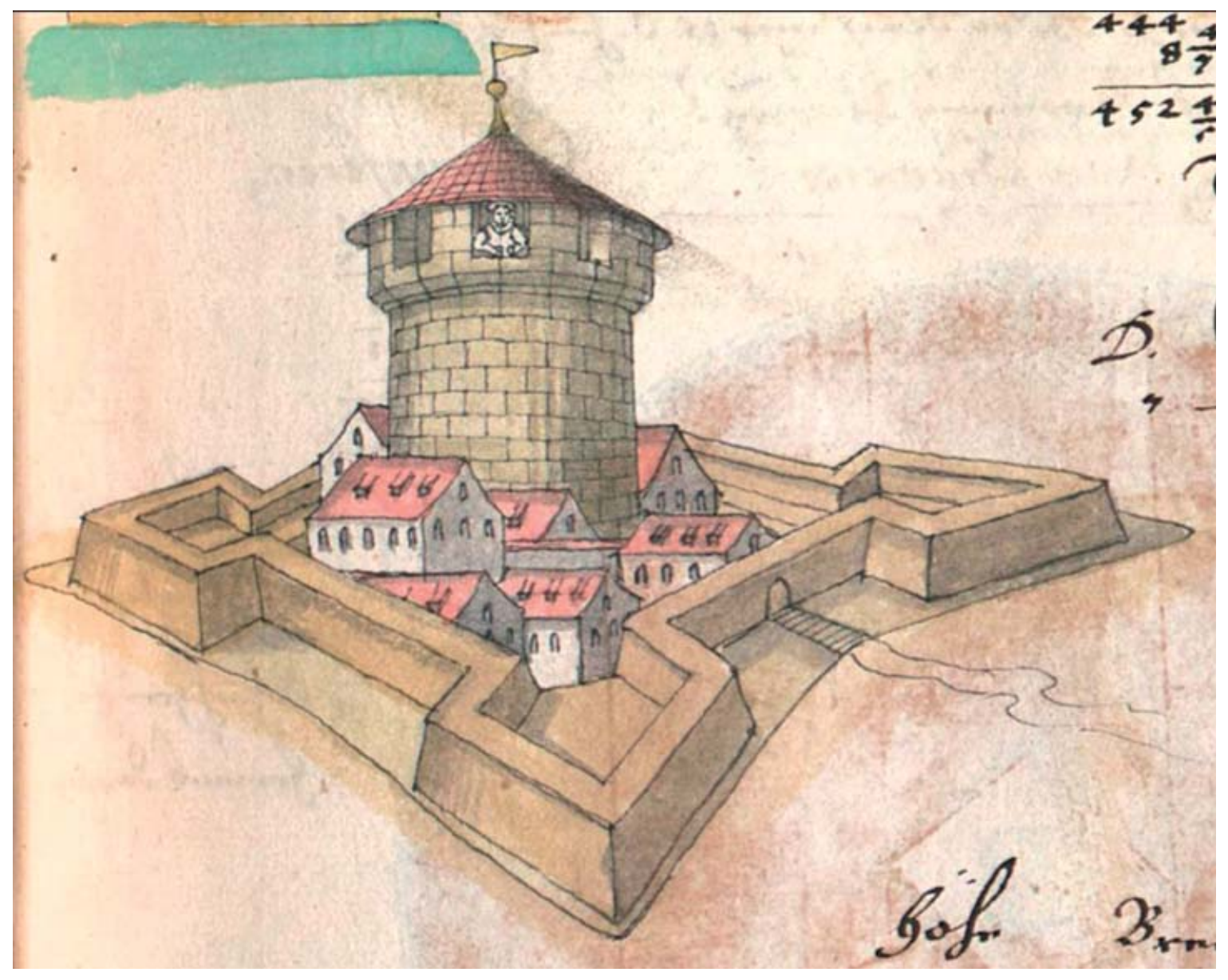

[Diese und vorangehende Abbildung:] Wendlers Planskizze und dekorative Zeichnung (in unterschiedlichen Maßstäben) zu den ,Materialkosten eines Turms' (Wendler, Neudörffers Grosse Arithmetic, Bayerische Staatsbibliothek München, Cgm 3789, 121r).

Aus den Maßen eines Turms und den Größen- und Preisangaben eines Mauersteins sind die Materialkosten des Turms zu bestimmen. Mit Außendurchmesser 6o Schuh, Innendurchmesser 24 Schuh und Höhe 112 Schuh ergibt sich das Mauervolumen (Differenz von Gesamtvolumen minus freiem Raum im Innern) zu 266.005 Schuh ${ }^{3}$. Ein Mauerstein habe ein Volumen von 27/4 Schuh ${ }^{3}$. Ohne Rücksicht auf Füllmaterial oder eventuelles Beschlagen der Steine wird das Mauervolumen näherungsweise durch das Volumen eines Steins geteilt, und man erhält die Anzahl der Steine mit 39.424. Da ein Mauerstein 55 Kreuzer kostet und ein Gulden 6o Kreuzern entspricht, kostet der Turm gut 36000 Gulden.

2016 eine Ausstellung von Rechenbüchern aus ihrem umfangreichen Bestand. Hinzu tritt eine von Studierenden der TH Nürnberg erarbeitete Online-Ausstellung www.regensburger-rechenmeister.de, die auf den Forschungsergebnissen in dem von uns herausgegebenen Band Erzählen und Rechnen in der frühen Neuzeit basiert.
Edith Feistner und Alfred Holl (Hrsg.): Erzählen und Rechnen in der frühen Neuzeit. Interdisziplinäre Blicke auf Regensburger Rechenbücher. Unter redaktioneller Mitarbeit von Jenny Huber und Nina Prifling. Berlin/Münster 2016.

Edith Feistner: Erstes Staatsexamen für Lehramt am Gymnasium Deutsch und Französisch, Dr. Phil. an der Ludwig-Maximilians-Universität München, Habil. an der Julius-Maximilians-Universität Würzburg. Lehrtätigkeit im Fach Germanistische Mediävistik an den Universitäten München, Augsburg, Würzburg und Kassel. Seit 2000 Inhaberin des Lehrstuhls für Germanistische Mediävistik an der Universität Regensburg (Literatur und Kultur des Mittelalters, u. a. mit Schwerpunkt historische Narratologie).

Alfred Holl: Diplom in Mathematik, Promotion in Romanischer Sprachwissenschaft an der Universität Regensburg, zusätzlich Studien in Computerlinguistik, Mathematikgeschichte, Paläographie, Klassischer Philologie, Indogermanischer und Vergleichender Sprachwissenschaft. Software-Entwickler und IT-Unternehmensberater. Seit 1990 Professor für Wirtschaftsinformatik an der Technischen Hochschule Nürnberg, Gastprofessuren in Schweden. Seit 2005 diverse Veröffentlichungen zu Themen der Mathematikgeschichte.

Prof. Dr. Edith Feistner, Institut für Germanistik, Universität Regensburg, Universitätsstraße 31, 93053 Regensburg edith.feistner@sprachlit.uni-regensburg.de

Prof. Dr. Alfred Holl, Fakultät Informatik, Technische Hochschule Nürnberg, Keßlerplatz 12, 90489 Nürnberg alfred.holl@th-nuernberg.de 